\title{
To Study the Effect of Selected Parameters on Adoption of Electronic Payment through Application in Selected Region of Aurangabad District
}

\author{
Pooja A. Kulkarni ${ }^{1}$, Dr. S. A .Ghumare ${ }^{2}$ \\ ${ }^{1}$ Assistant Professor, NBN Sinhgad School of Computer Studies, Pune, Maharashtra, India \\ ${ }^{2}$ Research Guide, Dr. B. A. M. University Aurangabad, Maharashtra, India
}

\begin{abstract}
Todays era is an IT era specifically human being depend on computer system for performing their daily routines (Needs) which include from reading newspaper to purchasing of vegetables or foods the banking operations is not an exception for that but while performing such operations human being heisted little bit because money involved in such an operations. This hesitation may leads to not using those services over internet but there are such parameters because of which user attracts for performing the financial transaction over cell phone few of them are user friendliness, convinces, security, platform etc cell phone is an device which user carry 24 by 7 in their pocket because of that user connect with the internet at any moment of time as well as because of latest technology like bootstrap web application develop in such a way that any layman can use those application more smartly. The UI of application very user friendly and attract many users to test the application. This paper is testing the impact of few parameters over the adoption of electronic payment though application. The primary assumptions of researcher is these parameters are responsible for performing the financial transaction by using electronic payment.
\end{abstract}

Keywords: Security, Convinces, Platform, User Friendliness, Authentication

\section{Introduction}

Today's era is era of Information Technology where Information has been provided on figure tip and many devices like Laptop, Palmtop, cell phone, desktop etc. provide those information to user. Software developers has develop many application and make attractive and informative GUI to provide convinces to user to use those application. These application also provide financial transaction. Financial transaction may include E-Commerce. M- Commerce, banking operations, fund transfer, wallets etc.

Today's user are net addict user for every transaction he/she relay in internet like finding nearest route to booking a date with friend, as well as transferring fund to friend or buying gift, in such transaction money has been involved and when there is enrolment of wealth user become conscious, to understand his consciousness to become hassle free transaction over internet many researchers as well as developers of these application provide many parameters, the researcher studying few parameters like security, convinces, platform, user friendliness, authentication in selected region of Aurangabad district.

\section{Literature Review}

Ms.Vaishnavi.J.Deshmukh, Sapna.S.Kaushik and Mr. Amit.M.Tayade has published article International Journal of Emerging Research in Management \&Technology Journal Title of article is "Payment Processing Systems and Security for E-Commerce: A Literature Review" Electronic Commerce is process of doing business through computer networks. A person sitting on his chair in front of a computer can access all the facilities of the Internet to buy or sell the products. Unlike traditional commerce that is carried out physically with effort of a person to go \& get products, ecommerce has made it easier for human to reduce physical work and to save time. E-Commerce which was started in early 1990's has taken a great leap in the world of computers, but the fact that has hindered the growth of e-commerce is security. Security is the challenge facing e-commerce today $\&$ there is still a lot of advancement made in the field of security, convinces, application development etc.

\section{Research Methodology}

According to Clifford Woody research comprises "defining and redefining problems, formulating hypothesis or suggested solutions collecting, organizing and evaluating data; making deduction and reaching conclusions and at last carefully testing the conclusions to determine whether they fit the formulating hypothesis."

Data was collected through survey from 30 users located in various geographical location of Aurangabad Region

1. Primary Data

2. Secondary Data

Primary Data: - Primary data are the data which are original in character, obtained for the first time, being collected from the users of net banking, either through questionnaire or through interviews via E-mail. This can be collected by various methods like

- Surveys

- Observation

- Questionnaires

Secondary data: -

Various sources of secondary data are Catalogues, Brochures, Magazines and Websites, Television etc. 


\section{International Journal of Science and Research (IJSR) \\ ISSN (Online): 2319-7064}

Index Copernicus Value (2013): 6.14 | Impact Factor (2015): 6.391

\section{Objectives}

1) To discuss Security, Convenience, user friendliness, authentication and platform do not differ significance between male and female users.

2) To discuss Security, Convenience, user friendliness, authentication and platform do not differ significance between rural and urban users.

3) To discuss Security, Convenience, user friendliness, authentication and platform do not differ significance between different age groups of users.

\section{Hypothesis}

1) Security, Convenience, user friendliness, authentication and platform do not differ significantly between male and female users.

2) Security, Convenience, user friendliness, and authentication do not differ significantly between rural and urban users

3) Platform do not differ significantly between rural and urban users

4) User friendliness and platform do not differ significantly among age groups of users

5) Security, Convenience, and authentication do not differ significantly among age groups of users

\section{Data Analysis and Interpretation}

\section{T-Test}

\begin{tabular}{|c|c|c|c|c|c|}
\hline \multicolumn{7}{|c|}{ Group Statistics } \\
\hline \multirow{3}{*}{ Security } & Gender & $\mathrm{N}$ & Mean & $\begin{array}{c}\text { Std. } \\
\text { Deviation }\end{array}$ & $\begin{array}{c}\text { Std. } \\
\text { Error Mean }\end{array}$ \\
\cline { 2 - 6 } & Male & 21 & 2.2857 & 1.18924 & .25951 \\
\hline & Female & 9 & 1.6667 & .50000 & .16667 \\
\hline Connivance & Male & 21 & 2.7143 & 1.61688 & .35283 \\
\cline { 2 - 6 } & Female & 9 & 3.3333 & .50000 & .16667 \\
\hline User friendliness & Male & 21 & 2.8571 & 1.27615 & .27848 \\
\cline { 2 - 6 } & Female & 9 & 3.0000 & .00000 & .00000 \\
\hline Puthentication & Male & 21 & 2.2857 & 1.52128 & .33197 \\
\cline { 2 - 6 } & Female & 9 & 2.0000 & .86603 & .28868 \\
\hline Platform & Male & 21 & 2.7143 & 1.61688 & .35283 \\
\cline { 2 - 6 } & Female & 9 & 3.6667 & 1.32288 & .44096 \\
\hline
\end{tabular}

\begin{tabular}{|c|c|c|c|c|c|c|c|c|c|c|}
\hline \multicolumn{11}{|c|}{ Independent Samples Test } \\
\hline & & \multicolumn{2}{|c|}{$\begin{array}{l}\text { Levene's Test } \\
\text { for Equality of } \\
\text { Variances }\end{array}$} & \multicolumn{7}{|c|}{ t-test for Equality of Means } \\
\hline & & \multirow[b]{2}{*}{$\mathrm{F}$} & \multirow[b]{2}{*}{ Sig. } & \multirow[b]{2}{*}{$\mathrm{T}$} & \multirow[b]{2}{*}{ df } & \multirow{2}{*}{$\begin{array}{c}\text { Sig. } \\
\text { (2-tailed) }\end{array}$} & \multirow{2}{*}{$\begin{array}{c}\text { Mean } \\
\text { Difference }\end{array}$} & \multirow{2}{*}{$\begin{array}{l}\text { Std. Error } \\
\text { Difference }\end{array}$} & \multicolumn{2}{|c|}{$\begin{array}{l}95 \% \text { Confidence Interval } \\
\text { of the Difference }\end{array}$} \\
\hline & & & & & & & & & Lower & Upper \\
\hline \multirow[t]{2}{*}{ Security } & Equal variances assumed & 6.045 & .020 & 1.494 & 28 & .146 & .61905 & .41435 & -.22971 & 1.46781 \\
\hline & Equal variances not assumed & & & 2.007 & 27.995 & .054 & .61905 & .30842 & -.01273 & 1.25083 \\
\hline \multirow[t]{2}{*}{ Connivance } & Equal variances assumed & 25.857 & .000 & -1.116 & 28 & .274 & -.61905 & .55475 & -1.75539 & .51730 \\
\hline & Equal variances not assumed & & & -1.586 & 26.609 & .124 & -.61905 & .39021 & -1.42025 & .18216 \\
\hline \multirow{2}{*}{$\begin{array}{c}\text { User } \\
\text { friendliness }\end{array}$} & Equal variances assumed & 17.157 & .000 & -.332 & 28 & .742 & -.14286 & .42970 & -1.02307 & .73735 \\
\hline & Equal variances not assumed & & & -.513 & 20.000 & .614 & -.14286 & .27848 & -.72376 & .43804 \\
\hline \multirow{2}{*}{$\begin{array}{l}\text { Authenticati } \\
\text { on }\end{array}$} & Equal variances assumed & 4.311 & .047 & .525 & 28 & .604 & .28571 & .54443 & -.82950 & 1.40093 \\
\hline & Equal variances not assumed & & & .649 & 25.389 & .522 & .28571 & .43993 & -.61963 & 1.19106 \\
\hline \multirow[t]{2}{*}{ Platform } & Equal variances assumed & .916 & .347 & -1.554 & 28 & .132 & -.95238 & .61300 & -2.20806 & .30329 \\
\hline & Equal variances not assumed & & & -1.686 & 18.491 & .109 & -.95238 & .56474 & -2.13661 & .23184 \\
\hline
\end{tabular}

H0: Security, Convenience, user friendliness, authentication and platform do not differ significantly between male and female

H1: Security, Convenience, user friendliness, authentication and platform differ significantly between male and female

Since, $\mathrm{p}>0.05$, we accept null hypothesis and conclude that Security, Convenience, user friendliness, authentication and platform do not differ significantly between male and female
T-Test

\begin{tabular}{|c|c|c|c|c|c|}
\hline \multicolumn{6}{|c|}{ Group Statistics } \\
\hline & Location & $\mathrm{N}$ & Mean & $\begin{array}{c}\text { Std. } \\
\text { Deviation }\end{array}$ & $\begin{array}{c}\text { Std. Error } \\
\text { Mean }\end{array}$ \\
\hline \multirow{2}{*}{ Security } & Urban & 24 & 2 & 0.88465 & 0.18058 \\
\hline & Rural & 6 & 2.5 & 1.64317 & 0.67082 \\
\hline \multirow{2}{*}{ Connivance } & Urban & 24 & 2.75 & 1.51083 & 0.3084 \\
\hline & Rural & 6 & 3.5 & 0.54772 & 0.22361 \\
\hline \multirow{2}{*}{ User friendliness } & Urban & 24 & 2.875 & 1.191 & 0.24311 \\
\hline & Rural & 6 & 3 & 0 & 0 \\
\hline \multirow{2}{*}{ Authentication } & Urban & 24 & 2.125 & 1.2959 & 0.26452 \\
\hline & Rural & 6 & 2.5 & 1.64317 & 0.67082 \\
\hline \multirow{2}{*}{ Platform } & Urban & 24 & 2.5 & 1.35133 & 0.27584 \\
\hline & Rural & 6 & 5 & 0 & 0 \\
\hline
\end{tabular}

Volume 5 Issue 6, June 2016 www.ijsr.net 
International Journal of Science and Research (IJSR)

ISSN (Online): 2319-7064

Index Copernicus Value (2013): 6.14 | Impact Factor (2015): 6.391

\begin{tabular}{|c|c|c|c|c|c|c|c|c|c|c|}
\hline \multicolumn{11}{|c|}{ Independent Samples Test } \\
\hline & & \multicolumn{2}{|c|}{\begin{tabular}{|c|} 
Levene's Test \\
for Equality of \\
Variances \\
\end{tabular}} & \multicolumn{7}{|c|}{ t-test for Equality of Means } \\
\hline & & \multirow[t]{2}{*}{$\mathrm{F}$} & \multirow[t]{2}{*}{ Sig. } & \multirow[t]{2}{*}{$\mathrm{T}$} & \multirow[t]{2}{*}{ df } & \multirow[t]{2}{*}{$\begin{array}{c}\text { Sig. } \\
\text { (2-tailed) }\end{array}$} & \multirow[t]{2}{*}{$\begin{array}{c}\text { Mean } \\
\text { Difference }\end{array}$} & \multirow[t]{2}{*}{$\begin{array}{l}\text { Std. Error } \\
\text { Difference }\end{array}$} & \multicolumn{2}{|c|}{$\begin{array}{l}95 \% \text { Confidence } \\
\text { Interval of the } \\
\text { Difference }\end{array}$} \\
\hline & & & & & & & & & Lower & Upper \\
\hline \multirow{2}{*}{ Security } & Equal variances assumed & 11.2 & 0.002 & -1.033 & 28 & 0.311 & -0.5 & 0.48412 & -1.49168 & 0.49168 \\
\hline & Equal variances not assumed & & & -0.72 & 5.744 & 0.5 & -0.5 & 0.6947 & -2.21838 & 1.21838 \\
\hline \multirow{2}{*}{ Connivance } & Equal variances assumed & 7.953 & 0.009 & -1.183 & 28 & 0.247 & -0.75 & 0.63387 & -2.04842 & 0.54842 \\
\hline & Equal variances not assumed & & & -1.969 & 23.572 & 0.061 & -0.75 & 0.38093 & -1.53696 & 0.03696 \\
\hline \multirow{2}{*}{$\begin{array}{c}\text { User } \\
\text { friendliness }\end{array}$} & Equal variances assumed & 8.547 & 0.007 & -0.254 & 28 & 0.802 & -0.125 & 0.49269 & -1.13423 & 0.88423 \\
\hline & Equal variances not assumed & & & -0.514 & 23 & 0.612 & -0.125 & 0.24311 & -0.62791 & 0.37791 \\
\hline \multirow{2}{*}{ Authentication } & Equal variances assumed & 2.426 & 0.131 & -0.602 & 28 & 0.552 & -0.375 & 0.62276 & -1.65067 & 0.90067 \\
\hline & Equal variances not assumed & & & -0.52 & 6.641 & 0.62 & -0.375 & 0.72109 & -2.09898 & 1.34898 \\
\hline \multirow{2}{*}{ Platform } & Equal variances assumed & 14.632 & 0.001 & -4.472 & 28 & 0 & -2.5 & 0.55902 & -3.64509 & -1.35491 \\
\hline & Equal variances not assumed & & & -9.063 & 23 & 0 & -2.5 & 0.27584 & -3.07062 & -1.92938 \\
\hline
\end{tabular}

H0: Security, Convenience, user friendliness, and authentication do not differ significantly between rural and urban users

H1: Security, Convenience, user friendliness, and authentication differ significantly between rural and urban users

Since, $\mathrm{p}>0.05$, we accept null hypothesis and conclude that Security, Convenience, user friendliness, and authentication do not differ significantly between rural and urban users H0: Platform do not differ significantly between rural and urban users

H1: Platform differ significantly between rural and urban users

Since, $p<0.05$, we reject null hypothesis and conclude that platform differ significantly between rural and urban users

\section{Oneway}

\begin{tabular}{|c|c|c|c|c|c|c|}
\hline \multicolumn{7}{|l|}{ ANOVA } \\
\hline & & Sum of Squares & Df & Mean Square & $\mathrm{F}$ & Sig. \\
\hline \multirow[t]{3}{*}{ Security } & Between Groups & 17.100 & 3 & \multirow{3}{*}{$\begin{array}{c}5.700 \\
.600\end{array}$} & \multirow[t]{3}{*}{9.500} & \multirow[t]{3}{*}{.000} \\
\hline & Within Groups & 15.600 & 26 & & & \\
\hline & Total & 32.700 & 29 & & & \\
\hline \multirow[t]{3}{*}{ Connivance } & Between Groups & 18.300 & 3 & \multirow{3}{*}{$\begin{array}{l}6.100 \\
1.477\end{array}$} & \multirow[t]{3}{*}{4.130} & \multirow[t]{3}{*}{.016} \\
\hline & Within Groups & 38.400 & 26 & & & \\
\hline & Total & 56.700 & 29 & & & \\
\hline \multirow[t]{3}{*}{ User friendliness } & Between Groups & 3.600 & 3 & \multirow{3}{*}{$\begin{array}{l}1.200 \\
1.119\end{array}$} & \multirow[t]{3}{*}{1.072} & \multirow[t]{3}{*}{.378} \\
\hline & Within Groups & 29.100 & 26 & & & \\
\hline & Total & 32.700 & 29 & & & \\
\hline \multirow{3}{*}{ Authentication } & Between Groups & 23.700 & 3 & \multirow{3}{*}{$\begin{array}{l}7.900 \\
1.119\end{array}$} & \multirow[t]{3}{*}{7.058} & \multirow[t]{3}{*}{.001} \\
\hline & Within Groups & 29.100 & 26 & & & \\
\hline & Total & 52.800 & 29 & & & \\
\hline \multirow[t]{3}{*}{ Platform } & Between Groups & 17.400 & 3 & \multirow{3}{*}{$\begin{array}{l}5.800 \\
2.100\end{array}$} & \multirow[t]{3}{*}{2.762} & \multirow[t]{3}{*}{.062} \\
\hline & Within Groups & 54.600 & 26 & & & \\
\hline & Total & 72.000 & 29 & & & \\
\hline
\end{tabular}

H0: User friendliness and platform do not differ significantly among age groups of users

H1: User friendliness and platform differ significantly among age groups of users
Since, $p>0.05$, we accept null hypothesis and conclude that User friendliness and platform do not differ significantly among age groups of users 


\section{International Journal of Science and Research (IJSR) ISSN (Online): 2319-7064}

Index Copernicus Value (2013): 6.14 | Impact Factor (2015): 6.391

Post Hoc Tests

\begin{tabular}{|c|c|c|c|c|c|c|c|c|}
\hline \multicolumn{9}{|c|}{ Multiple Comparisons } \\
\hline \multirow{2}{*}{\multicolumn{2}{|c|}{ Dependent Variable }} & \multirow[b]{2}{*}{ (I) Age } & \multirow[b]{2}{*}{ (J) Age } & \multirow{2}{*}{$\underset{(\mathrm{I}-\mathrm{J})}{\text { Mean Difference }}$} & \multirow{2}{*}{ Std. Error } & \multirow{2}{*}{ Sig. } & \multicolumn{2}{|c|}{ 95\% Confidence Interval } \\
\hline & & & & & & & Lower & Upper \\
\hline \multirow{12}{*}{ Security } & \multirow{12}{*}{ LSD } & \multirow{3}{*}{ 18-24Years } & 24-30 Years & -0.6 & 0.37417 & 0.121 & -1.3691 & 0.1691 \\
\hline & & & 30-36 Years & $1.40000^{*}$ & 0.37417 & 0.001 & 0.6309 & 2.1691 \\
\hline & & & Above 36 Years & $1.40000^{*}$ & 0.4899 & 0.008 & 0.393 & 2.407 \\
\hline & & \multirow{3}{*}{ 24-30 Years } & 18-24Years & 0.6 & 0.37417 & 0.121 & -0.1691 & 1.3691 \\
\hline & & & 30-36 Years & $2.00000^{*}$ & 0.44721 & 0 & 1.0807 & 2.9193 \\
\hline & & & Above 36 Years & $2.00000^{*}$ & 0.54772 & 0.001 & 0.8741 & 3.1259 \\
\hline & & \multirow{3}{*}{ 30-36 Years } & 18-24Years & $-1.40000^{*}$ & 0.37417 & 0.001 & -2.1691 & -0.6309 \\
\hline & & & 24-30 Years & $-2.00000^{*}$ & 0.44721 & 0 & -2.9193 & -1.0807 \\
\hline & & & Above 36 Years & 0 & 0.54772 & 1 & -1.1259 & 1.1259 \\
\hline & & \multirow{3}{*}{$\begin{array}{c}\text { Above } 36 \\
\text { Years }\end{array}$} & 18-24Years & $-1.40000^{*}$ & 0.4899 & 0.008 & -2.407 & -0.393 \\
\hline & & & 24-30 Years & $-2.00000^{*}$ & 0.54772 & 0.001 & -3.1259 & -0.8741 \\
\hline & & & 30-36 Years & 0 & 0.54772 & 1 & -1.1259 & 1.1259 \\
\hline \multirow{12}{*}{ Convenience } & \multirow{12}{*}{ LSD } & \multirow{3}{*}{ 18-24Years } & 24-30 Years & -0.8 & 0.58704 & 0.185 & -2.0067 & 0.4067 \\
\hline & & & 30-36 Years & $-1.80000^{*}$ & 0.58704 & 0.005 & -3.0067 & -0.5933 \\
\hline & & & Above 36 Years & $-1.80000^{*}$ & 0.76862 & 0.027 & -3.3799 & -0.2201 \\
\hline & & \multirow{3}{*}{ 24-30 Years } & 18-24Years & 0.8 & 0.58704 & 0.185 & -0.4067 & 2.0067 \\
\hline & & & 30-36 Years & -1 & 0.70165 & 0.166 & -2.4423 & 0.4423 \\
\hline & & & Above 36 Years & -1 & 0.85934 & 0.255 & -2.7664 & 0.7664 \\
\hline & & \multirow{3}{*}{ 30-36 Years } & 18-24Years & $1.80000^{*}$ & 0.58704 & 0.005 & 0.5933 & 3.0067 \\
\hline & & & 24-30 Years & 1 & 0.70165 & 0.166 & -0.4423 & 2.4423 \\
\hline & & & Above 36 Years & 0 & 0.85934 & 1 & -1.7664 & 1.7664 \\
\hline & & \multirow{3}{*}{$\begin{array}{c}\text { Above } 36 \\
\text { Years }\end{array}$} & 18-24Years & $1.80000^{*}$ & 0.76862 & 0.027 & 0.2201 & 3.3799 \\
\hline & & & 24-30 Years & 1 & 0.85934 & 0.255 & -0.7664 & 2.7664 \\
\hline & & & 30-36 Years & 0 & 0.85934 & 1 & -1.7664 & 1.7664 \\
\hline \multirow{12}{*}{ Authentication } & \multirow{12}{*}{ LSD } & \multirow{3}{*}{ 18-24Years } & 24-30 Years & $-1.10000^{*}$ & 0.51103 & 0.041 & -2.1504 & -0.0496 \\
\hline & & & 30-36 Years & $1.40000^{*}$ & 0.51103 & 0.011 & 0.3496 & 2.4504 \\
\hline & & & Above 36 Years & $1.40000^{*}$ & 0.6691 & 0.046 & 0.0246 & 2.7754 \\
\hline & & \multirow{3}{*}{ 24-30 Years } & 18-24Years & $1.10000^{*}$ & 0.51103 & 0.041 & 0.0496 & 2.1504 \\
\hline & & & 30-36 Years & $2.50000^{*}$ & 0.6108 & 0 & 1.2445 & 3.7555 \\
\hline & & & Above 36 Years & $2.50000^{*}$ & 0.74807 & 0.003 & 0.9623 & 4.0377 \\
\hline & & \multirow{3}{*}{ 30-36 Years } & 18-24Years & $-1.40000^{*}$ & 0.51103 & 0.011 & -2.4504 & -0.3496 \\
\hline & & & 24-30 Years & $-2.50000^{*}$ & 0.6108 & 0 & -3.7555 & -1.2445 \\
\hline & & & Above 36 Years & 0 & 0.74807 & 1 & -1.5377 & 1.5377 \\
\hline & & & 18-24Years & $-1.40000^{*}$ & 0.6691 & 0.046 & -2.7754 & -0.0246 \\
\hline & & $\begin{array}{l}\text { Above } 36 \\
\text { Years }\end{array}$ & 24-30 Years & $-2.50000^{*}$ & 0.74807 & 0.003 & -4.0377 & -0.9623 \\
\hline & & & 30-36 Years & 0 & 0.74807 & 1 & -1.5377 & 1.5377 \\
\hline
\end{tabular}

H0: Security, Convenience, and authentication do not differ significantly among age groups of users

H1: Security, Convenience, and authentication differ significantly among age groups of users

Since, $p<0.05$, we reject null hypothesis and conclude that Security, Convenience, and authentication differ significantly among age groups of users.

Post hoc test revealed that,

- Security in age groups 18-24Years and 24-30 Years differ significantly from age groups 30-36 Years, Above 36 Years

- Convenience in age group 18-24Years, differ significantly from age groups 30-36 Years, Above 36 Years

- Authentication in age groups 18-24Years and 24-30 Years differ significantly from age groups 24-30 Years, 30-36 Years, Above 36 Years and 18-24Years, 30-36 Years, Above 36 Years respectively.

\section{Conclusion}

The security, convenience, user friendliness, authentication and platform are the major parameters for adoption of electronic parameter through payment among which platform (Application Deployment / user interface) differs significantly between rural and urban users that means platform is major parameter on which Service provider has to think upon.

\section{References}

\section{Research Papers \& Journals}

[1] Kirui, O. K. (n.d.). Impact of mobile phone-based money transfer services in agriculture: evidence from Kenya. International Association of Agricultural Economists (IAAE)

[2] Ms.Vaishnavi.J.Deshmukh， Sapna.S.Kaushik and Mr. Amit.M.Tayade has published article International

\section{Volume 5 Issue 6, June 2016 www.ijsr.net}

Licensed Under Creative Commons Attribution CC BY 


\section{International Journal of Science and Research (IJSR) \\ ISSN (Online): 2319-7064}

Index Copernicus Value (2013): 6.14 | Impact Factor (2015): 6.391

Journal of Emerging Research in Management \&Technology Journal Title of article is " Payment Processing Systems and Security for E-Commerce: A Literature Review"

[3] International Journal of Engineering, Business and Enterprise Applications (IJEBEA), Comparitive Study of Online Banking Security System of various Banks in India Rajpreet Kaur Jassal1 , Dr. Ravinder Kumar Sehgal2 1Computer Science \& Engineering, BBSBEC Fatehgarh Sahib Punjab, INDIA. 2 Principal, JSSIET Kauli Patiala, Punjab,INDIA.

[4] Journal of Electronic Commerce Research, VOL. 3, NO. 1, 2002 Page 15 SECURITY CONTROL IN INTER-BANK FUND TRANSFER Dan Zhu Department of Logistics, Operations and MIS Iowa State University

[5] International Journal on Recent and Innovation Trends in Computing and Communication ISSN: 2321-8169 Volume: 3 Issue: 2 391- 398.

[6] Analysis of User Authentication Methods \& Impact on Identification Especially in Banking Abdul Samad Shaikh Department of Computer Science \& IT GreenfoTech Education, Aurangabad.

[7] http://ageconsearch.umn.edu/bitstream/126624/2/Owuor. pdf

\section{Books}

[8] Research Methodology Methods and Techniques C. R. Kothari

[9] Wireless and Mobile Network Security -HakimaChaouchiMaryline Laurent-Maknavicius 